\title{
Combined effects of frequency and layer removal on background track characteristics of ECE polycarbonate detectors
}

Mehdi Sohrabi*, Zahra Soltani, Amir Hakimi

Health Physics and Radiation Dosimetry Research Laboratory, Department of Energy Engineering and Physics, Amirkabir University of Technology, Tehran, Iran

\begin{abstract}
Polycarbonate track detectors (PCTD) when electrochemically etched (ECE) provide excellent characteristics for registering relatively lower-LET charged particles (e.g. alphas, fast-neutroninduced recoils) for many health physics and ion detection applications. The layer removal method of PCTDs by ethylenediamine (EDA) developed in our laboratory reduces the background track (BGT) density significantly. The frequency of the applied electric field strongly affects the BGTdensity and diameter and thus affects the minimum detection limit (MDL). In order to study the combined effects of the frequency and layer removal on the BGT density and thus on the MDL, this research was conducted. The BGT density versus the layer thickness removed at frequencies up to $12 \mathrm{kHz}$ decrease rapidly to about 10-20 $\mu \mathrm{m}$ above which they reach aminimum constantlevel, while the mean BGT diameter verses layer removed at all frequencies are constant with flat responses. On the other hand the BGT density and diameter versus frequency at different layers removed up to $\sim 50 \mu \mathrm{m}$ increase till $4 \mathrm{kHz}$ above which they reach plateaus. The PCTDs with $\sim 20 \mu \mathrm{m}$ layer removal at frequencies up 1 to $2 \mathrm{kHz}$ showed the lowest MDL. The results are presented and discussed.
\end{abstract}

*Corresponding Author; Mehdi Sohrabi, E-mail; dr_msohrabi@yahoo.com

Keywords: Polycarbonate, ECE, background, frequency, layer removal, MDL 


\section{Introduction}

The electrochemical etching (ECE) of charged particle tracks in polymer track detectors has been the subject of research and development as well as applications for over 4 decades (Tommasino and Armellini, 1973, Sohrabi, 1974, 1975). The ECE method is governed by a large number of chemical and physical parameters in particular the applied electric field strength and frequencywhich have been under some theoretical and experimental studies (Tommasino and Armellini, 1973, Sohrabi, 1974, 1975, 1980, 1985, 1986, Wong and Tommasino, 1982, Hassib and Piesch, 1978, Olszewski et al., 1982, Boyang, 1984, Singh and Virk, 1987, Sohrabi et al. 2012). Some frequency studies have been focused on polycarbonate track detectors (PCTD) (Sohrabi, 1975, 1980, Wong and Tommasino, 1987, Singh and Virk, 1987, Hussain and Khan, 1988). Wong and Tommasino (1982) concluded that the power dissipation is an important factor on the alpha-exposed PCTDs up to $1 \mathrm{MHz}$ using Maxwell-Wagner interfacial polarization theory. Olszewski et al. (1982) studied the vibration of LR-115 under a forced harmonic oscillation to justify a "resonance-type effect" in frequency responses. Boyang (1984) reported the electrostriction in ECE and measured the vibration of CR-39 foils by the Michelson interference (He-Ne laser). Sohrabi $(1975,1980)$ showed a resonance-type track density versus frequency response at $2 \mathrm{kHz}$ and later reported discovery of an internal heating effect in the ECE of the PCTDs and CR-39 using small ECE chambers (Sohrabi, 1985,1986). This internal heating effect was discussed to be due to polymer dielectric loss with a rate which increases as the frequency increases proportional to the square of field strength,dielectric constant $(\dot{\varepsilon})$ and dielectric loss tangent ( $\tan \delta$ ) of the polymer as well as reporting the observation of electrostriction, electro-osmosis and electrophoresis (Sohrabi, 1985,1986). Singh and Virk (1987) concluded that the ECE should be done at a field frequency where $\varepsilon$ and $\tan \delta$ is a maximum to raise the temperature to increase the registration efficiency. Hussein and Khan (1991) studied the ECE of ${ }^{252} \mathrm{Cf}$ fission fragment tracks at a fix field strength at frequencies 0.1$10.0 \mathrm{kHz}$ confirming an efficiency peak at $2 \mathrm{kHz}$ the same as concluded by Sohrabi (1975, 1980).

One of the main obstacles of polymer track detectors in general and when processed by the ECE in particular is the background track (BGT) density at different electric field frequencies. The BGT density highly affects the minimum detection limit (MDL) for low-dose or low-fluence 
charged particle detection and dosimetry applications. The BGT density may be originated from the inherent polymer defects and radon and daughters' alpha tracks depending on the age of the PCTDs and possible scratches. The BGT density also varies from polymer to polymer; in the same polymer from batch to batch as well as due to handling care, environmental conditions, aging, manufacturer, ECE parameters and in particular the applied field conditions. The BGT density reduction method based on layer removal from the PCTD's surface by ethylenediamine (EDA) solutions has been highly effective for PCTDs processed by ECE at $2 \mathrm{kHz}-\mathrm{HV}$ (Dadvand and Sohrabi, 1998) and by $50 \mathrm{~Hz}-\mathrm{HV}$ field conditions (Sohrabi et al. 2014a). The frequency of the applied electric field also plays a major roleon the track registration response including the BGT density and diameter. Therefore, BGT characteristics studies at different frequencies are of high importance for low-dose or low-fluence charged particle detection applications which directly affects the MDL. It is the purpose of this research to study:

1. the effects of the layer removed from the surface of $250 \mu \mathrm{m}$ thick PCTDs prior to the ECE process at different frequencies up to $12 \mathrm{kHz}$ on BGT density and diameter,

2. the effects of frequency for different layer removals on BGT density and diameter in the PCTDs, and

3. the combined effects of the frequency and the layer removal of the PCTDs on the MDL at different confidence levels.

\section{Material and methods}

Polycarbonate track detectors (PCTDs) used were $250 \mu \mathrm{m}$ thick and $3 \mathrm{~cm} \times 3 \mathrm{~cm}$ in size cut from larger sheets with masks on both sides to prevent scratches and BGT induction. The masks of the sides were removed before the layer removal process. Care was taken not to develop any scratches on the surface of the PCTD. EDA solutions of $70 \%$ concentration in water at $26^{\circ} \mathrm{C}$, as proved highly efficient for layer removal from previous studies without affecting the surface qualities of the PCTDs (Dadvand and Sohrabi, 1998, Sohrabi et al., 2014), was used for the layer removal. Prior to the ECE process of the PCTDs at each frequency, the PCTDs were subjected to 10, 20, 30, 40 and $50 \mu \mathrm{m}$ thick layer removal. The layer removed from the PCTD was determined by a micrometer with a measurement range of 0 to $25 \mathrm{~mm}$ with $1 \mu \mathrm{m}$ accuracy.

After the layer removal process and drying of the PCTDs, they were processed by the ECE method by applying $800 \mathrm{~V}$ at a frequency range of 1 to $12 \mathrm{kHz}$. The PCTDs were placed 
between the two semi-chambers of the triplet ECE chamber (Sohrabi, 1993); the side to be etched was filled with PEW solution $\left(15 \mathrm{~g} \mathrm{KOH}+40 \mathrm{~g} \mathrm{C}_{2} \mathrm{H}_{5} \mathrm{OH}+45 \mathrm{~g} \mathrm{H}_{2} \mathrm{O}\right)$ at $26{ }^{\circ} \mathrm{C}$ and the side not to be etched was filled with $3.3 \%$ hydrochloric acid. The mean BGT density and mean diameter were determined under a light microscope coupled to a CDC device with a computer. All the background tracks on a PCTD were scanned for the total area with a total magnification 40x; i.e. 10x ocular and $4 \mathrm{x}$ objective.

One of the parameters of importance to be considered for accurate measurements of low dose or low charge particle fluences is the MDL which is highly dependent on the BGT density. To determine the MDL with a confidence level, the statistical method advanced by Altshuler and Pasternack (1963) was applied in this study. The results obtained well match with those of similar methods applied by German Standard DIN 25482 (1989), Cember and Johnson (2009) and Dimitrova et al. (2011) as reported by Sohrabi et al. (2014a).

\section{Results and discussion}

\subsection{Effects of layer removal on BGT characteristics}

The ECE-processed PCTDs like any solid state nuclear track detector have a BGT density which highly affects the MDL of the detector for low-dose low-fluence charged particle measurements, if the BGT density is not adequately treated or taken into proper account. Figures 1 and 2 show the mean BGT density and mean track diameter respectively as functions of the layer removal thickness from the surface of the PCTDs for electric field conditions of $800 \mathrm{~V}$ at different frequencies from 1 to $12 \mathrm{kHz}$. It should be noted that for each layer removal, one PCTD was used and processed by the ECE method after the layer removal. 


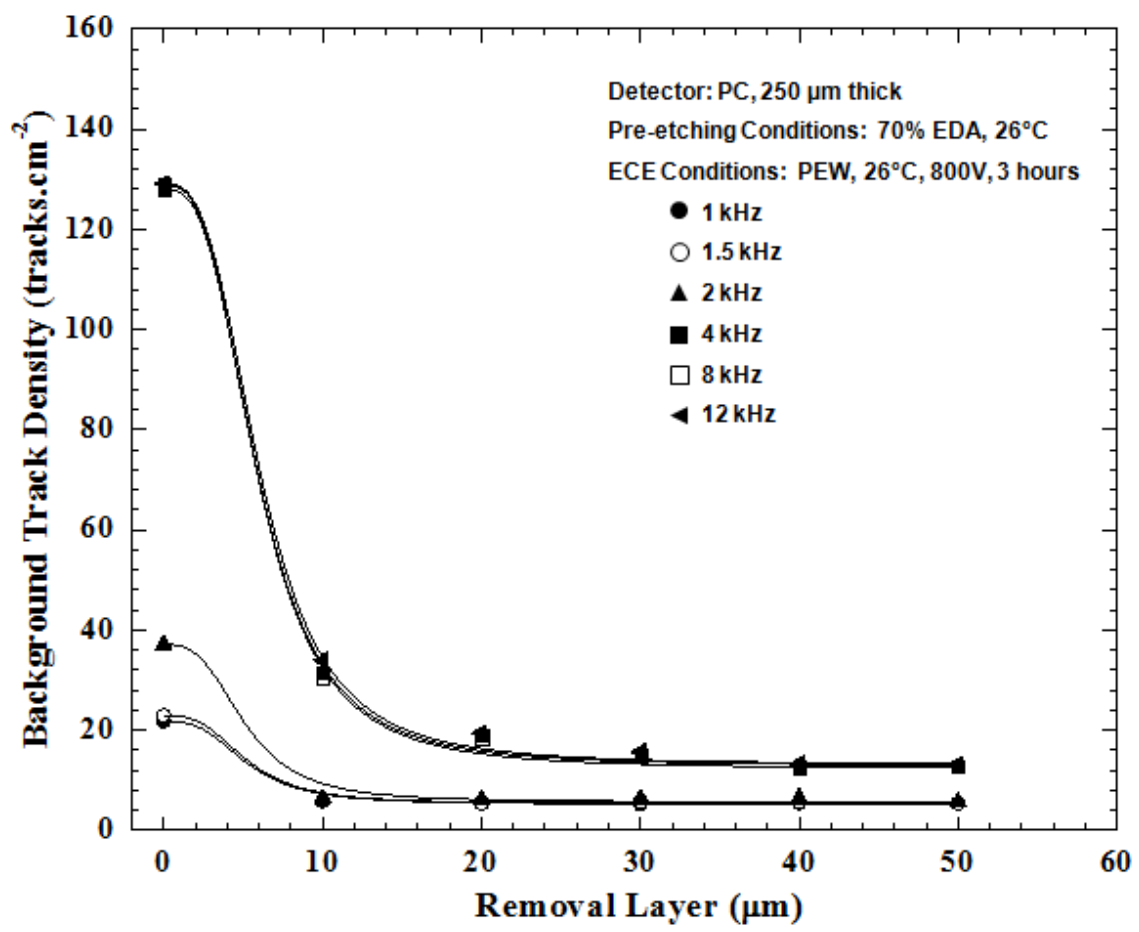

Fig. 1. Mean BGT density (tracks. $\mathrm{cm}^{-2}$ ) versus removed layer at different ECE frequencies at the stated ECE conditions. 


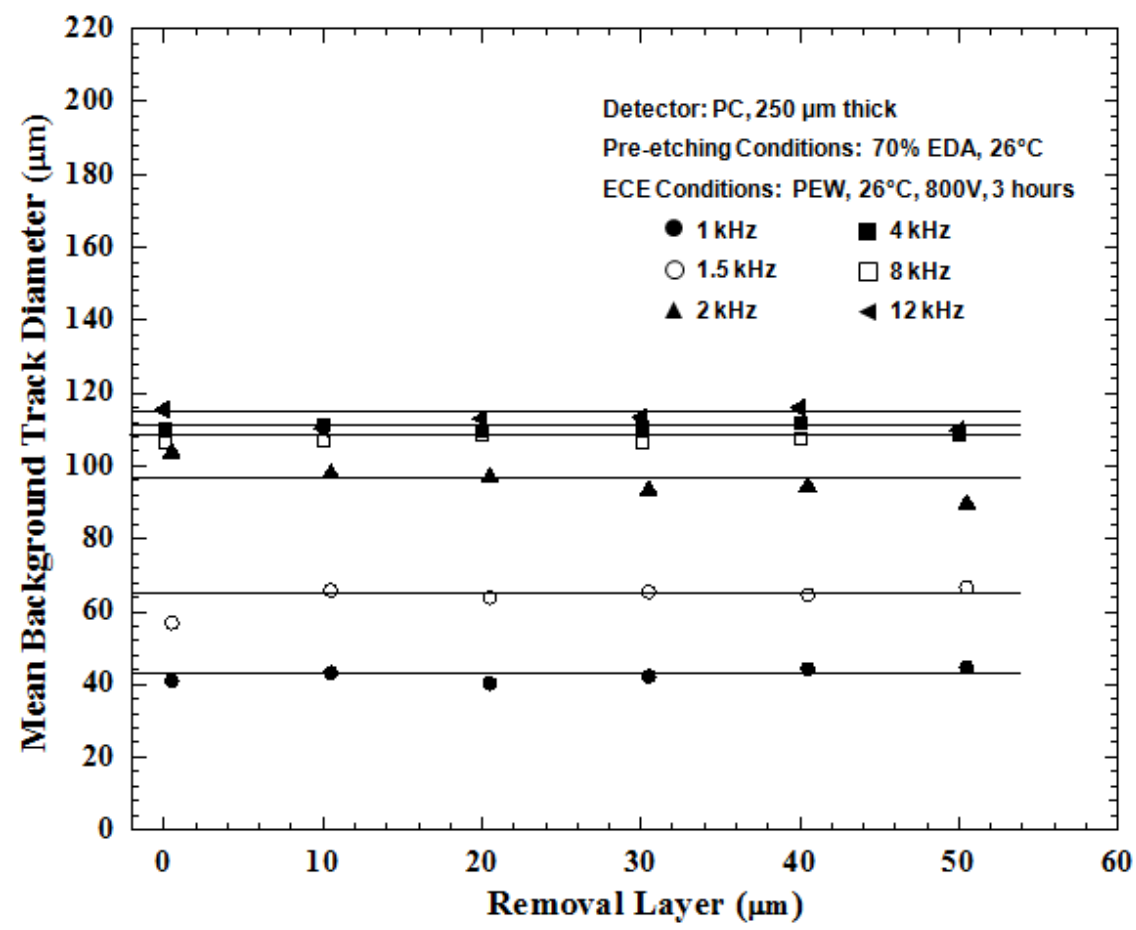

Fig. 2. Mean BGT diameter versus removed layer at different ECE frequencies at the stated ECE conditions.

As it can be observed from Figure 1, the mean BGT density in PCTDs with no layer removal has the highest values of $21.7,22.9,37.1,128.8,127.9$ and 129.1 tracks.cm $^{-2}$ for frequencies $1,1.5$, 2, 4, 8 and $12 \mathrm{kHz}$ respectively. After the layer removal, the BGT densities are reduced rapidly reaching minimum values after 10 to $20 \mu \mathrm{m}$ layer removal and the values stay almost constant, the level of which depends on the frequency. On the other hand, as seen in Figure 2, the mean BGT diameter is frequency dependent. At a fixed frequency however the mean BGT diameter stays constant for each PCTD having a specific layer removal. This implies that the layer removal process, no matter what the thickness of the layer removal is, did not change the surface characteristics of the detector for low-fluence or low-dose applications such as neutron dosimetry and environmental radon measurements.

\subsection{Effectsof frequency on BGTcharacteristics}

The mean BGT density and mean diameter as functions of applied ECE frequency for PCTDs with different fixed layers removed from the surface are shown in Figures 3 and 4. As it can be seen, as the frequency increases for a PCTD with a fixed removed layer, the mean BGT 
density and mean diameter increase as the frequency increases up to about $4 \mathrm{kHz}$ after which the density and diameter stay constant.

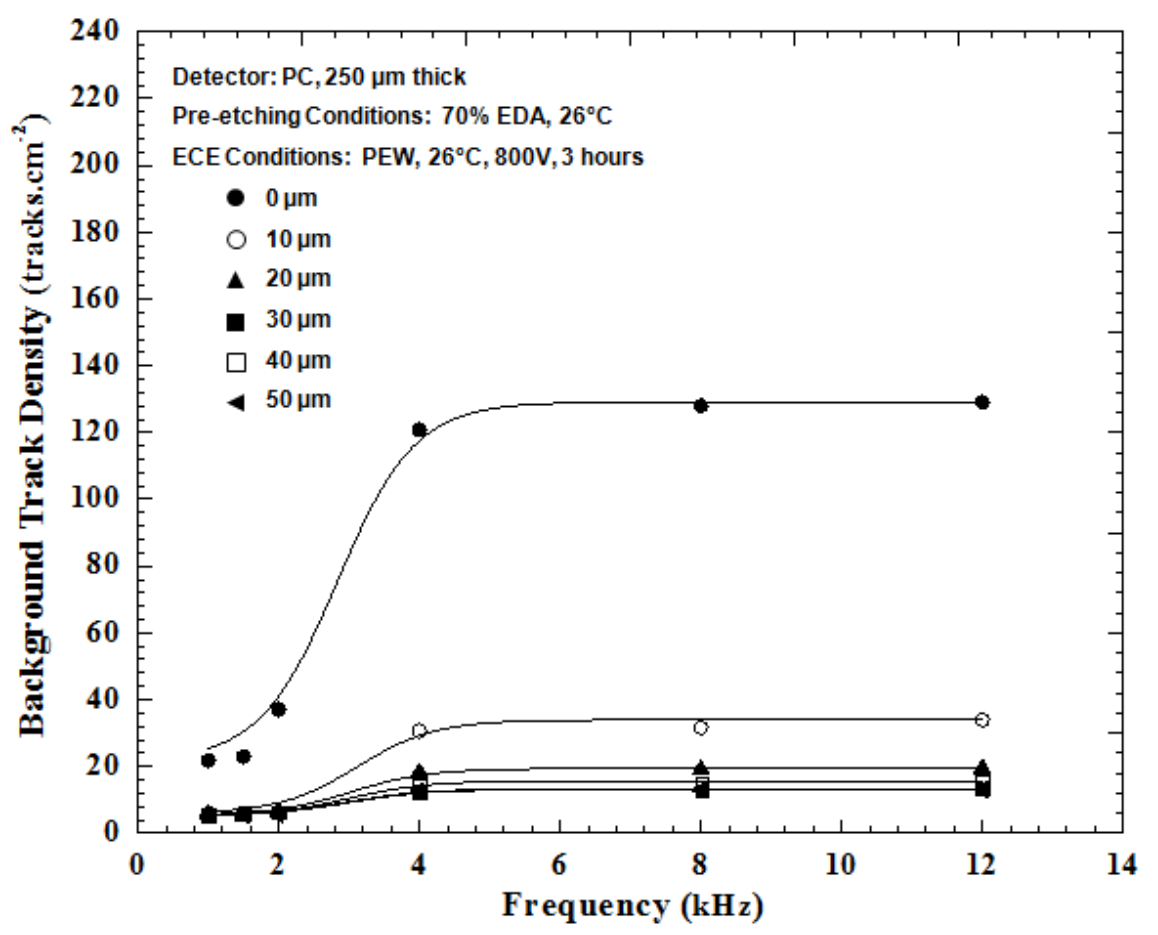

Fig. 3. Mean BGT density (tracks.cm ${ }^{-2}$ ) versus frequency for PCTDs with different layers removed from the surface at the stated ECE conditions. 


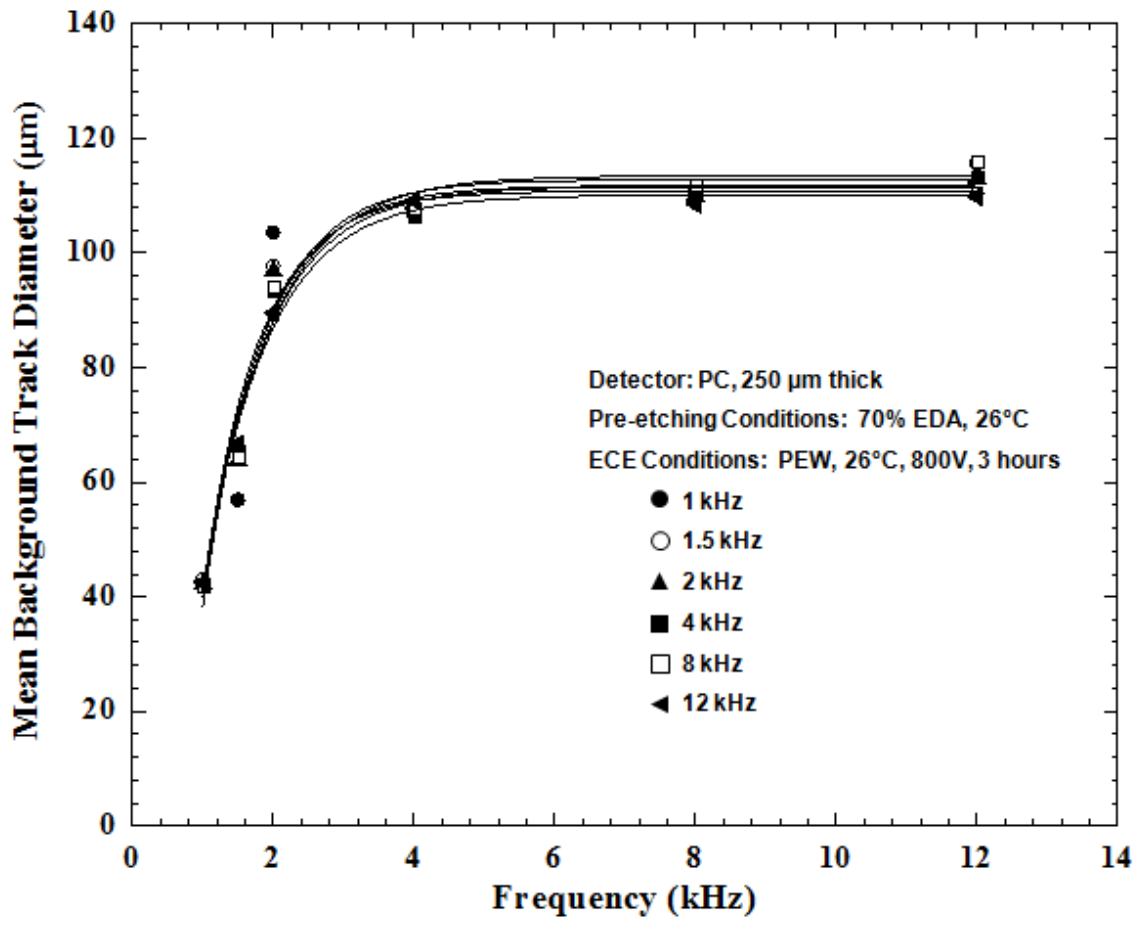

Fig. 4. Mean BGT diameter $(\mu \mathrm{m})$ versus frequency for different removed layers at the stated ECE conditions.

As it can be seen in Figure 3, as the frequency increases the BGT density also increases with a rate which is more pronounced with no layer removal than that of when a layer is removed. In fact, with a layer removal, the increase of BGT density versus frequency is less than that of when a thin layer below $10 \mu \mathrm{m}$ is removed. For example, at $4 \mathrm{kHz}$ frequency when no layer is removed, the BGT density is 6 times higher than that of $1 \mathrm{kHz}$, while with only $10 \mu \mathrm{m}$ layer removed, the density at $4 \mathrm{kHz}$ is only 1.5 times higher. This supports further the efficiency of the layer removal method for BGT density reduction. As another example, the BGT density with no layer removal at $12 \mathrm{kHz}$ is about 130 tracks. $\mathrm{cm}^{-2}$. When $30 \mu \mathrm{m}$ is removed, this density will be reduced to only 15 tracks. $\mathrm{cm}^{-2}$ which is about 9 times smaller thus reducing the MDL of the PCTD significantly for low-dose low-fluence charged particle applications.

It seems the observed effects of the frequency on the BGT characteristics can be related to some phenomena as discussed above (Tommasino and Armellini, 1973, Sohrabi, 1975, 1980, 1985, 1986, Wong and Tommasino, 1982, Hassib and Piesch, 1978, Sohrabi et al. 2012, Olszewski et al., 1982, Boyang, 1984, Singh and Virk, 1987). As shown in Figure 3, by increasing the frequency for each thickness removed, the BGT density and diameter increase especially when no layer removal has been performed. This observation can be correlated to 
"internal heating rate" or heat generated per unit volume of the polymer during ECE process and in particular the local heating at the track site being under electrostriction which is proportional to the square of the field strength, frequency (f) and polymer loss index or loss factor (Sohrabi,1985,1986). Wong and Tommasino (1982) have also concluded that the increase in the track density and diameter of charge particle tracks as frequency increases can be correlated to the increase in power loss of the polymer. So, the "internal heating effect" governed by parameters such as the polymer loss index or loss factor, in which frequency plays an important role, can be responsible for increasing the BGT density and diameter.

\subsection{Effects of frequency and layer removal on $M D L$}

The MDL is the lowest quantity of track density or dose that can be statistically distinguished from the BGT density within a desired confidence level. The MDL is highly dependent on the BGT density which is affected by the applied ECE field conditions (Sohrabi and Ramezani, 2014, Sohrabi et al. 2014b). As stated in the materials and methods section above, the MDL at different conditions applied was determined by the statistical method advanced by Altshuler and Pasternack (1963) and tested in comparison with methods applied by others (German Standard DIN 25482,1989, Cember and Johnson, 2009 and Dimitrova et al., 2011). Details on the statistical method applied was described before (Sohrabi et al., 2014a).

Table 1 shows the MDL values calculated at different confidence levels for varying frequencies and removed layers. The mean BGT density when no layer is removed is 21.7, 22.9, $37.1,128.8,127.9$ and 129.1 tracks.cm ${ }^{-2}$ for 1.0, 1.5, 2.0, 4.0, 8.0 and $12 \mathrm{kHz}$ respectively, as also stated above. Accordingly, the MDL with 99.9\% level of confidence calculated are 49.7, 51.6, 73.6, 196.5, 195.8 and 197.3 tracks.cm ${ }^{-2}$ for the mentioned frequencies respectively. For 90.0\% level of confidence, the MDL values are 33.7, 35.1, 52.6, 157.9, 156.9 and 158.2. Such values for confidence levels $99.0,97.5$ and $95 \%$ lie between the two sets of values for the $99.9 \%$ and $90 \%$. 
Table 1. MDL at different confidence levels for different frequencies and removed layers for 250 $\mu \mathrm{m}$ thick PCTD with layer removal in $70 \%$ EDA at $26^{\circ} \mathrm{C}$.

\begin{tabular}{|c|c|c|c|c|c|c|c|}
\hline $\begin{array}{l}\text { Frequency } \\
\quad(\mathrm{kHz})\end{array}$ & $\begin{array}{c}\text { Removed } \\
\text { Layer } \\
(\mu \mathrm{m})\end{array}$ & $\begin{array}{c}\text { BGT } \\
\text { Density }\end{array}$ & $\begin{array}{c}99.9 \% \\
\text { confidence } \\
\text { level }\end{array}$ & $\begin{array}{c}99 \% \\
\text { confidence } \\
\text { level }\end{array}$ & $\begin{array}{c}97.5 \% \\
\text { confidence } \\
\text { level }\end{array}$ & $\begin{array}{c}95 \% \\
\text { confidence } \\
\text { level }\end{array}$ & $\begin{array}{c}90 \% \\
\text { confidence } \\
\text { level }\end{array}$ \\
\hline \multirow{6}{*}{1} & 0 & 21.7 & 49.72 & 43.47 & 40.02 & 37.03 & 33.68 \\
\hline & 10 & 5.9 & 20.42 & 17.17 & 15.38 & 13.83 & 12.08 \\
\hline & 20 & 5.6 & 19.91 & 16.73 & 14.97 & 13.45 & 11.74 \\
\hline & 30 & 5.2 & 18.91 & 15.85 & 14.16 & 12.70 & 11.05 \\
\hline & 40 & 5.4 & 19.39 & 16.27 & 14.55 & 13.06 & 11.38 \\
\hline & 50 & 5.31 & 19.14 & 16.05 & 14.34 & 12.87 & 11.21 \\
\hline \multirow{6}{*}{1.5} & 0 & 22.9 & 51.61 & 45.20 & 41.66 & 38.60 & 35.15 \\
\hline & 10 & 6.0 & 20.61 & 17.34 & 15.53 & 13.97 & 12.21 \\
\hline & 20 & 5.3 & 19.07 & 15.99 & 14.29 & 12.82 & 11.16 \\
\hline & 30 & 5.7 & 20.02 & 16.83 & 15.06 & 13.53 & 11.81 \\
\hline & 40 & 5.6 & 19.80 & 16.63 & 14.88 & 13.36 & 11.66 \\
\hline & 50 & 5.4 & 19.34 & 16.23 & 14.51 & 13.02 & 11.35 \\
\hline \multirow{6}{*}{2} & 0 & 37.1 & 73.59 & 65.43 & 60.92 & 57.03 & 52.64 \\
\hline & 10 & 6.3 & 21.34 & 17.98 & 16.12 & 14.52 & 12.71 \\
\hline & 20 & 6.1 & 20.94 & 17.63 & 15.80 & 14.22 & 12.44 \\
\hline & 30 & 6.3 & 21.34 & 17.98 & 16.12 & 14.52 & 12.71 \\
\hline & 40 & 6.5 & 21.71 & 18.30 & 16.42 & 14.80 & 12.97 \\
\hline & 50 & 5.6 & 19.78 & 16.61 & 14.86 & 13.34 & 11.64 \\
\hline \multirow{6}{*}{4} & 0 & 128.8 & 196.94 & 181.73 & 173.34 & 166.07 & 157.90 \\
\hline & 10 & 30.6 & 63.77 & 56.36 & 52.27 & 48.73 & 44.75 \\
\hline & 20 & 18.2 & 43.80 & 38.08 & 34.92 & 32.19 & 29.12 \\
\hline & 30 & 14.6 & 37.51 & 32.39 & 29.56 & 27.12 & 24.37 \\
\hline & 40 & 12.3 & 33.34 & 28.64 & 26.05 & 23.80 & 21.28 \\
\hline & 50 & 12.8 & 34.19 & 29.41 & 26.76 & 24.48 & 21.90 \\
\hline \multirow{6}{*}{8} & 0 & 127.9 & 195.82 & 180.66 & 172.29 & 165.05 & 156.91 \\
\hline & 10 & 31.6 & 65.30 & 57.77 & 53.61 & 50.01 & 45.97 \\
\hline & 20 & 19.0 & 45.17 & 39.33 & 36.10 & 33.31 & 30.17 \\
\hline & 30 & 14.9 & 38.04 & 32.87 & 30.02 & 27.55 & 24.77 \\
\hline & 40 & 12.8 & 34.28 & 29.49 & 26.84 & 24.55 & 21.97 \\
\hline & 50 & 12.9 & 34.41 & 29.60 & 26.95 & 24.65 & 22.07 \\
\hline \multirow{6}{*}{12} & 0 & 129.1 & 197.27 & 182.05 & 173.64 & 166.37 & 158.19 \\
\hline & 10 & 33.9 & 68.85 & 61.05 & 56.74 & 53.01 & 48.82 \\
\hline & 20 & 19.4 & 45.74 & 39.85 & 36.59 & 33.78 & 30.61 \\
\hline & 30 & 15.6 & 39.37 & 34.07 & 31.14 & 28.61 & 25.76 \\
\hline & 40 & 13.3 & 35.09 & 30.21 & 27.52 & 25.19 & 22.57 \\
\hline & 50 & 13.1 & 34.78 & 29.93 & 27.26 & 24.94 & 22.34 \\
\hline
\end{tabular}


As an example, Figure 5 shows the graphical trend that the MDL versus the removed layer for different frequencies vary for the MDL with $99.9 \%$ confidence level when no layer is removed. After about $20 \mu \mathrm{m}$ layer removed at any frequency, the MDL reaches a minimum value which depends on the frequency. The data for other confidence levels also follow the same graphical trends as those of Figure 5 but of course with different values.

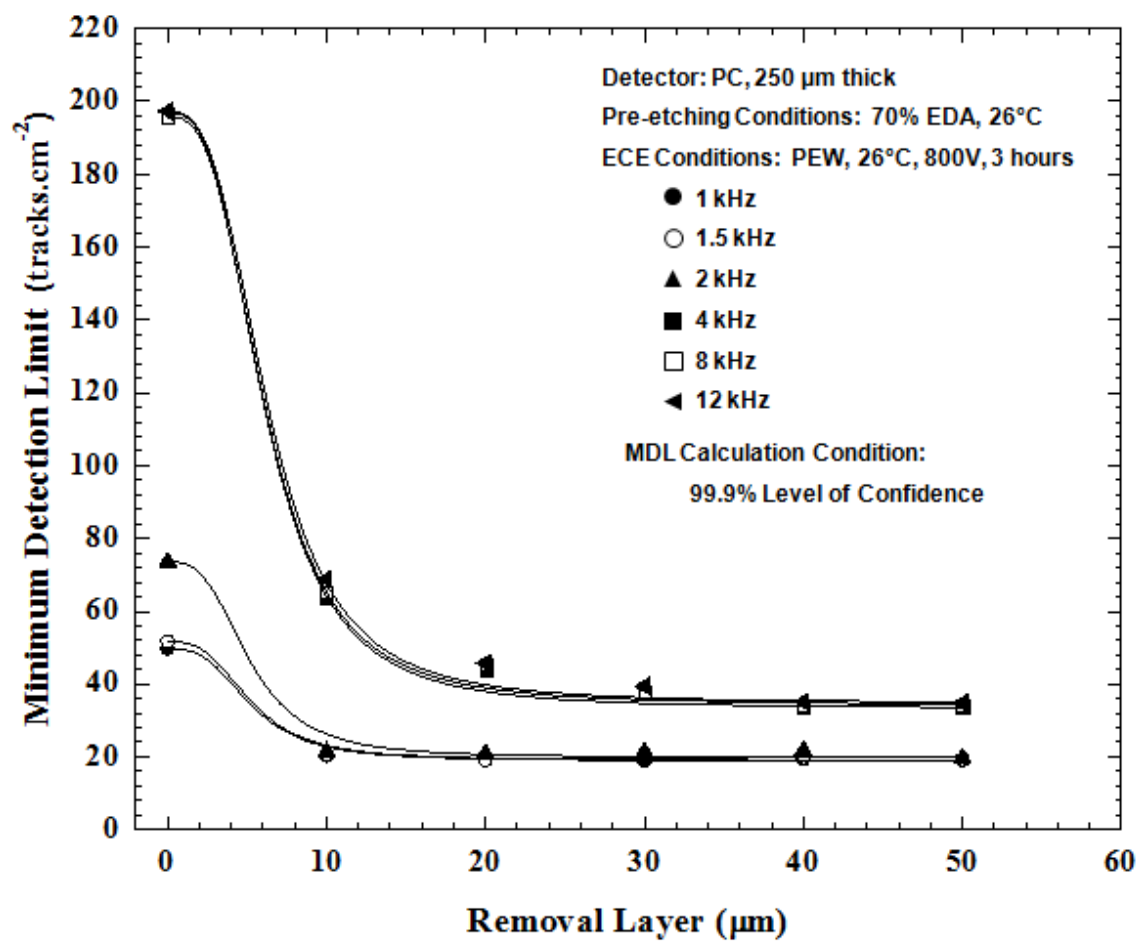

Fig. 5. MDL (99.9\% confidence level) versus removed layer at different field frequencies.

\section{Conclusions}

The combined effects of the frequency and the layer removal from the surface of the $250 \mu \mathrm{m}$ thick PCTDs on the BGT density and diameter and on the MDL at different confidence levels were studied. Some conclusions follow:

- The frequency of the electric field applied to the PCTD, when other ECE parameters are fixed, play an important role on the BGT density and diameter and in turn on the MDL. As the frequency increases, the mean BGT density and diameter increases up to $4 \mathrm{kHz}$ after which they reach plateaus.

- The layer removal method is highly effective for the BGT density reduction to reduce the MDL without changing the surface track registration characteristics (Sohrabi et al, 2014a). 
- The frequency responses as concluded can be correlated tothe "internal heating rate" or heath generated per unit volume of the PCTD under ECE process and in particular on the local heating at the track site being under electrostriction; it is proportional to the square of the field strength, frequency (f) and polymer loss index or loss factor.

- The MDL is frequency and layer removal dependent. It was decreased to the lowest MDL at minimum plateau levels after $\sim 20 \mu \mathrm{m}$ layer removed from the PCTD at frequencies up to 2 $\mathrm{kHz}$.

\section{Acknowledgement}

Research was conducted at the Department of Energy Engineering and Physics, Amirkabir University of Technology, under the current budget.

\section{References}

Altshuler, B., Pasternack, B., 1963. Statistical measures of the lower limit of detection of a radioactivity counter. J. Health Phys. 9, 293-298.

Boyang, Li. 1982. Electrostriction in electrochemical etching (ECE) and its effect on the formation of tree track in foil CR39. Nucl. Tracks and Radiat. Meas. 8.1: 105-108.

Cember, H., Johnson, T.E., 2008. Introduction to Health Physics, $4^{\text {th }}$ ed., McGraw Hill (ISBN 007-105461-8), 864 pages.

Currie, L.A., 1968. Limits for qualitative detection and quantitative determination: application to radiochemistry. Anal. Chem. 40(3), 586-593.

Dadvand, N., Sohrabi, M., 1998. A method for reducing background in plastic detectors.Appl Radiat Isotopes. 49, 1609-1611.

Dimitrova, I., Mitev, K., Pressyanov, D., Georgiev, S., Boshkova, T., 2011. Measurement of ${ }^{222} \mathrm{Rn}$ and ${ }^{226} \mathrm{Ra}$ in water by absorption of radon in polycarbonates and etching alphatracks. Radiat. Meas. 46(1), 119-126.

DIN 25482. 1989. Nachweisgrenze und Erkennungsgrenze bei Kernstrahlungsmessungen.

Hassib, M., Piesch, E., 1978. Electrochemical etching of neutron-induced tracks in plastic detectors using a $50 \mathrm{~Hz}$ electric supply.Nucl. Instrum. Meth. 154 (2), 377-381.

Hussain, G., Khan, H. A. 1991. Frequency response characteristics of electrochemically etched tracks in Makrofol-E polycarbonate track detectors. Nucl. Instrum. Methods Phys. Res. B. 61(2), 194-196. 
Olszewski, J., Domahski, T., Hawryitski, M., Chrukielewski, W., 1982. Some aspects and results of the electrochemical etching of a thin-foil track detector. Nucl. Tracks. 6.4, 161-174.

Singh, R. C., Virk, H. S., 1987. Internal heating effect during electrochemical etching of Lexan polycarbonate. Nucl. Instrum. Meth. Phys. Res. B. 29(3), 579-582.

Sohrabi, M., 1974. Electrochemical etching amplification of recoil particle tracks in polymers and its application in fast neutron personnel dosimetry. J. Health Phys. 27, 598-600.

Sohrabi, M., 1975. Electrochemical etching amplification of low LET recoil particle tracks for fast neutron dosimetry. Ph.D. Thesis, Ga. Inst. Tech. Atlanta, Georgia.

Sohrabi, M., 1980. Electrochemical etching of fast-neutron-induced recoil tracks: the effects of field strength and frequency. Nucl. Tracks. 4(3), 131-140.

Sohrabi, M., 1985. Discovery of an "internal heating effect" during electrochemical etching of polymeric dosimeters. Nucl. Instrum. Methods Phys. Res. A. 238(2), 517-523.

Sohrabi, M.,1986. Discovery of an "internal heating effect" during electrochemical etching of polymeric dosimeters: a study of polymer characteristics. Nucl. Tracks and Rad. Meas. 12, Nos. 1-6, 179-183.

Sohrabi, M. 1993. A new triplet electrochemical etching (TECE) method. Radiat. Prot. Dosim. 48(3), 279-283.

Sohrabi, M., Habibi, M., Roshani, G. H., Ramezani, V., 2012. A novel method for observation by unaided eyes of nitrogen ion tracks and angular distribution in a plasma focus device using $50 \mathrm{~Hz}-\mathrm{HV}$ electrochemically-etched polycarbonate detectors. Radiat. Meas. 47(7), 530-536.

Sohrabi, M., Hakimi, A., Soltani, Z., 2014a. Reducing inherent background track density of $1 \mathrm{~mm}$-thick polycarbonate detectors processed by $50 \mathrm{~Hz}-\mathrm{HV}$ ECE method. Oral Presentation. $8^{\text {th }}$ Int. Conf. on High Levels of Natural Radiation and Radon Areas. Book of Abstracts, p. 104, 1-5 September, Prague, Czech Republic.

Sohrabi, M., Ramezani,V., Habibi, M. 2014b. A new method for detection of alpha particles in $1 \mathrm{~mm}$ thick polycarbonate detectors using $50 \mathrm{~Hz}$ - HV ECE method. Radiat. Meas. 67, 5966.

Sohrabi, M., Ramezani, V. 2015. Alpha particle energy response of $1 \mathrm{~mm}$-thick polycarbonate track detectors by $50 \mathrm{~Hz}$ - HV electrochemical etching method. Radiat. Prot. Dosim. 164 (3), 244-251. 
Tommasino, L., Armellini, C., 1973. A new etching technique for damage track detectors. Radiat. Eff. 20(4), 253-255.

Wong, C. F., Tommasino, L., 1982. The frequency response of electrochemical etching. Nucl. Tracks and Radiat. Meas. 6(1), 25-34. 